\title{
LAYERS AND LEVELS: What a Column of Water Tells Us about Human Cognition
}

\author{
Wes Raykowski \\ Griffith University, Queensland, Australia \\ wes.rajkowski@griffithuni.edu.au
}

\begin{abstract}
The notion of levels can be found in many everyday expressions, such as top-level destination, entry-level sales, low-level panic, high risk level, basic-level research, high level of care, level of meaning, level of knowledge, level of freedom, and level of importance. I argue that these are metaphorical expressions in which the respective abstract concepts can be understood in terms of the more palpable experience of the levels to which we are accustomed through the handling of liquids. By looking at the interaction between SCALE and ITERATION image schemas, this article examines an embodied interpretation of levels, layers and water columns in the context of containers to facilitate a better understanding of these experiences and their use as a source domain for conceptual metaphors in language, science and mathematics. The conceptual analysis in this paper is limited to English expressions.
\end{abstract}

\section{Keywords}

conceptual metaphor - container - level - layer - embodied logic

\section{Introduction}

Ever since the publication of Metaphors We Live By (Lakoff and Johnson, 1980), a small book that introduced the idea of conceptual metaphors to the public at large, there has been renewed interest in the role of metaphors in communication. To date, hundreds if not thousands of conceptual metaphors have been studied in great detail. A metaphor that, to my knowledge, has not been explored (at least not to the extent that it deserves) is the notion of levels and, relatedly, layers. The term 'level' is often used in concrete situations in

(C) RAYKOWSKI, 2018 | DOI 10.1163/23526416-00401005

This is an open access article distributed under the terms of the prevailing CC-BY license at the time of publication. 
which the word is understood literally. A level can represent a horizontal, flat and smooth surface (or line), any point of which may or may not be at a fixed height or depth in reference to another surface or line (e.g., the level tracks of land, the bottom of a vessel, a horizontal position, the sea's surface, or the foot of a mountain). This literal meaning can also be found in the context of comparison (e.g., the levels in two vessels are the same), movement (e.g., flying at a certain elevation), and action (leveling off the ground or a hedge). When it is used to discuss abstract concepts, the term level imposes a specific framework in which the target concept is viewed as being stratified in a way that can be described by the experiential framework of the source domain. This imposition can be observed in phrases such as the level of linguistic theory, the level of current scholarship, and the level of analysis. Although none of these target notions (theory, scholarship, and analysis) resemble the flat surfaces of water in a container, a mountain plateau, or the position of an instrument, the term level seems to invoke the overall organization found in these and perhaps many other physical situations that serve as the source domain for the metaphors discussed in this paper.

The concept of levels is productive, as it can be used in combination in a great variety of contexts. For example, the term level can be, and often is, used in relation to sensations (e.g., level of pressure/lightness), emotions and feelings (e.g., levels of joy/fear), social relations (e.g., level of worth/control), engineering (e.g., pressure/volume levels), physics (e.g., level of measurement and energy levels), biology (e.g., cellular and ecological levels), geography (e.g., taxonomic levels and level of resolution), music (e.g., harmony/volume level), art (e.g., level of saturation/brightness), philosophy (e.g., levels of enquiry/ analysis), economics and politics (e.g., price and earnings levels), sociology (e.g., level of health/wellbeing), and linguistics (e.g., level of meaning/syntax). In this paper, I argue that these and many other conceptual metaphors that use the notion of levels are best understood in terms of the experience of handling water.

The objectives of the paper are not limited to the embodied description of levels and their use in metaphorical expressions, both of which are most likely widely accepted. The main contribution the article makes involves the conceptual interaction between levels and layers of a water column contained within a vessel. The interactions are discussed in the paper in terms of the SCALE and ITERATION image schemas, the first of which represents the concept of nesting (in this paper, nested levels) and the second the notion of repetition (in this paper, repetition of layers and containers). The choice of these specific image schemas is not accidental or arbitrary. They represent two distinct ways of thinking which co-occur in some situations. For example, magnitudes 
often occur together with multitudes, intensity with extent, value with range, degree with scope, pitch with duration, altitude with longitude, and quality with quantity - just as the notion of levels co-occur with layers. As in the case of all image schemas which are often difficult to grasp due to their unconscious origins (Evans and Green, 2006: 180), the interaction between the SCALE and ITERATION image schemas is not obvious until it is pointed to and described in sufficient detail - which is the main objective of this paper.

In the first subsection of the introduction, I discuss and justify the methodology adopted in the paper. Next, I argue that the handling of water in containers of human scale creates the best opportunity for observing changes in water levels. I then discuss the use of the container schema in linguistics. Section 2 is focused on the embodied experience associated with handling liquids. Its subsections utilize various diagrams and symbols extensively to ensure the economy and precision of the argument. The subsections address topics such as the issue of comparison and inequalities, adding and removing layers, the relationship between levels and layers, making layers identical, the conceptual duality of columns, idempotence, differentiating levels, and cognitive products and ways of representing them. The paper concludes with Section 3, which describes some of the consequences of the analysis in the preceding sections for cognitive metaphor theory and linguistics in general. The current paper is a detailed exploration of issues outlined in the book "Conceptual Understructure of Human Experience: Thesis" (Raykowski, 2014).

Please note that the conceptual analysis in this paper is limited to English language expressions and the concepts found in basic-level mathematics and physics.

\subsection{Methodological Issues}

Drafting a paper based on a framework that is unfamiliar to its reader presents a particular challenge. To be introduced to any new framework, the reader must reinterpret at least some held ideas, which is not possible without already being familiar with the proposed scheme. The use of diagrams is perhaps the best way to overcome this difficulty. Unfortunately, this approach is not without problems. Johnson cautions against the uncritical reliance on diagrams, especially in relation to image schemas (Johnson, 1987: 22-23). However, the fact that the diagrams Johnson refers to depict both the embodied experience on which a schema is based and the physical objects that are the source of this experience is often neglected. To understand image schemas and metaphors, one must have some knowledge of the objects on which they are based. For example, to correctly use the container schema, the linguist must recognize that content can strongly affect the experience of the container; for example, the containment of solid objects is not the same as that 
of gas, which differs from the experience of liquids. All such nuances can be easily depicted with diagrams. The use of diagrams in turn lends itself to symbolic expressions that make the embodied description more economical and precise. This paper pays particular attention to the objects of experience, the use of diagrams, and the symbolic notations upon which they are based. Without this shift in focus, it would not be possible to argue that the metaphorical use of levels is related to the common experience of handling water. The approach is problematic, however, as it creates a disconnect between the sections dedicated to language (Sections 1 and 3) and that on the properties of water columns (Section 2), which may appear to some readers as being unrelated to linguistics.

\subsection{Why Water?}

With the exception of the category of tools, there are basically two senses of the word level. One pertains to the object-like horizontal lines or surfaces that are not much different from the concept of layers. The other sense, which is related to the notions of height and depth, is the relationship between horizontal lines or planes, where one is used as a reference. To observe the peculiarity of the level concept, consider three sentences in which levels describe the intensity of emotions: "The study found that when jurors felt moderate to high levels of disgust, they also reported increased levels of anger" (Estrada-Reynolds et al, 2016: 353). "Younger people (18-25) have consistently reported lower levels of wellbeing than older Australians" (APS, 2015:4). "What are the reasons behind the high levels of happiness in Denmark?" (Wiking, 2014: 5). These and many other examples in the literature, both lay and scientific, demonstrate that the use of the idea of levels to express emotions and feelings is quite common. Surprisingly, humans have no difficulty differentiating between levels of emotional intensity but find it impossible to state precisely the extent to which they differ. This inability suggests that, unlike height, which is defined as an extent, levels represent an ordering system that does not depend on spatial relations and measurement. Furthermore, the sensations of intensity are private in the sense that no person other than their 'owner' can experience them. To express the level of intensity to others, the subject must point in some way (e.g., by referring) to the object the experience of which best 'conveys' a private feeling. In the context of cognitive metaphor theory, the object to which one points is called the source domain, and the private emotion is called the target domain.

\section{Source domain $\quad \rightarrow \quad$ Target domain}

$$
\text { (levels of water } \left.)_{\text {single unit }} \quad \rightarrow \quad \text { (levels of intensity }\right)_{\text {single individual }}
$$


As outlined above, the target domain has certain properties: the extent of intensity is limited to the single individual; thus, the source domain must be a unit capable of varying its levels internally. Pointing to a level line or plane (i.e., to a level track) is of little help because no single entity can express variation. Similarly, reference to the changing levels of a lake or sea also fails to be useful because the surface of such bodies is not always flat, and even when it is, the levels change imperceptibly. However, the experience of handling water in a container, even if it is just the palm of a hand shaped into a scoop, is not only universal but also as ancient as the human existence itself. Water is ubiquitous and essential to human life, and the combination of water and gravity was, for a long time, the main source of energy in many ancient societies. The issue of levels was critical for harnessing this energy, and water-based levels were often used for this purpose (Rossi, Russo and Russo, 2009). Today, the experience of handling water is even more readily available. From early childhood, the members of the speaking community are exposed to water and water-based mixtures in a variety of ways and situations. There is great assortment of containers, and many of them are transparent, which facilitates the observation of liquid levels.

In summary, human-scale containers (i.e., ones that can be handled by human beings) and small quantities of water (the surface of which is easy to stabilize) are the most likely (but not only) source of experience with changing levels. A column of water combines levels with layers in a surprisingly sophisticated way. Due to its unconscious nature, it is difficult to appreciate the richness of the experience related to handling water. One objective of this paper is to make this experience available to readers and show how it relates to linguistic expression. Before this objective can be achieved, however, the relationship between a container and its contents must be discussed in some detail.

\subsection{The Container Image Schema}

In a general sense, beyond complex configurations, there are three main combinations of containers and their contents: open containers that hold solid objects (Figure $1 \mathrm{~b}$ ), sealed containers that hold a gas (Figure 1c), and containers that hold liquids (Figure 1d). Most metaphors discussed in the contemporary literature refer to the containment of discrete objects. A typical diagram based on Johnson's interpretation of the container schema (1987: 32) shows a circle with a solid boundary and an arrow pointing to the outside of the circle (Figure 1a). To enter or leave the container, a part of its wall must be removed (Figure $1 \mathrm{~b}$ ). The presence of the opening means that both container A and objects $\mathrm{C}$ exist in the same space (shown in gray). This common space allows 


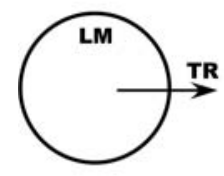

a

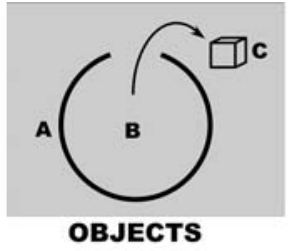

b

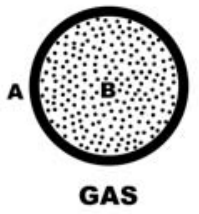

C

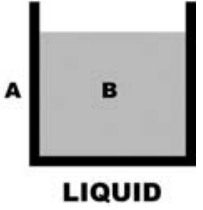

d

FIGURE 1 Container image schemas. The containment of solid objects is not the same as that of gas, which differs from the experience of liquids.

them to be moved in relation to each other. This type of container is used as a source domain for the metaphors in which the target is (or is treated as) a discrete entity: John went out of the room. Let out your anger. Pick out the best theory (Johnson, 1987: 32). In these sentences, the object-like content is moved into or out of the container.

However, this container-content combination is not suitable for metaphors involving intensity. To express intensity, one needs a combination capable of conveying variability of the type described by Mark Johnson as the SCALE image schema: "Scales have a cumulative character of a special sort. If you are collecting money and have accumulated \$15, then you also have \$10" (1987: 122). Thus, $\$ 15$ includes $\$ 10$, which includes $\$ 5$, and so on, which can be represented as an inequality $\$ 15>\$ 10>\$ 5>\$ 0$ or with nested brackets $(((())))$. The intensity of emotions is an example of the SCALE schema. In the case of anger, the sense of being very angry includes moderate anger, which in turn includes no anger. The pressures of gas and steam in a sealed container (Figure 1c) have identical properties. From personal experience, most readers know that when the pressure can no longer be contained, the container will blow violently apart. Unsurprisingly, the experience associated with pressure vessels is often used to express anger: He was bursting with anger. When I told him, he just exploded. I blew my stack. (Kövecses, 2010: 198). The pressure metaphor can be used to express a scalar order with modifiers, such as very high, high, average, low, very low and no (e.g., intensity); however, it cannot convey the extent to which those levels differ. As a result, they represent different experiences to different people.

The sensation of intensity can also be expressed with water levels but in a different way. Consider the following sentences in which feelings are interpreted as a liquid substance and intensity as the level of the liquid: She could feel her gorge rising. My anger kept building up inside me. When too much 
liquid is added, the container will overflow, and this image is used to express an excess of feelings: I brimmed over with joy when I saw her. She overflowed with joy. (Kövecses, 2010: 124-125) The process that underlies these examples is described by Kövecses as a correlation of recurrent experiences:

This [MORE IS UP] metaphor operates with two concepts: quantity and verticality. Quantity consists of a scale that has more and less, while verticality consists of one that has up and down (...) This is because the more specific correlation is that when the quantity or amount of a substance increases (more), the level of the substance rises (up) and when the quantity of the substance decreases (less), the level of the substance goes down (down). (Kövecses, 2010: 80)

This interpretation does not describe how the levels relate to the substance except that they go up/down when the substance is added or removed. The absence of this relationship makes the communication imprecise. Consider adding more soil to a heap of dirt. The notion of levels in this case is not very clear, and their relationship to the substance is rather complicated. However, adding an identical volume of water to a clear cylindrical container connects the layers of water with their levels in a predictable way. Such an arrangement combines quantity (interpreted here as a repetition of volume) with verticality (which represents the SCALE image schema) in a precise way: the levels are experienced as if they were separated by identical layers, which allows the expression of the relationship between levels in terms of layers. In other words, one can easily express private feelings (their levels) in terms of concrete and public objects (layers).

Please note that such expressions are only obliquely related to the process of measuring understood as a comparison of public entities of the same type. In the case of level metaphors, the intensity is intangible and private, and the layers are discrete, concrete and public. Such mapping is, therefore, a typical metaphorical expression that involves “(...) understanding and experiencing one kind of thing in terms of another" (Lakoff and Johnson, 1980:5). The same relationship can be found in other embodied experiences, such as slicing a loaf of bread, walking and perceiving temperature through the use of a thermometer scale. Therefore, $\underline{I}$ do not insist that the water column experience is the only source domain for level metaphors. The notion of levels and, relatedly, layers, is, in this view, the result of often unconscious experiences involving changes in levels in a great variety of situations. The next section describes the embodied experience based on folk and expert accounts of handling water. 
Cognitive metaphors are sometimes described as "(...) mappings between a more concrete or physical source domain and a more abstract target domain" (Kövecses, 2010: 77). The terms concrete and abstract have many meanings, but in this paper, they are defined entirely in terms of space considered in the context of handling water. Most readers will be intimately familiar with the act of pouring a liquid out of one vessel into another. Figure 2a depicts a situation in which a distinct volume of water in cup "a" is transferred into larger cylindrical vessel "b." The cup and the vessel are separated by physical space; in human experience, this makes them distinct and discrete, hence, concrete. However, when the water from the cup is poured into the vessel, the two volumes are no longer distinct. The pouring of water can be conceptualized as either bringing two objects together (in this case, volumes) or removing space from between the objects in a process referred to as concatenation. If space can be removed, then it can also be inserted through the process known as division. Therefore, the notion of space plays a critical role in defining concatenation, division, continuity, the distinctness of objects and their (spatial) relationship.

Figure $2 \mathrm{~b}$ illustrates another basic human experience: a clear vessel filled with liquids of different densities and color will form visible layers. If the bottom of the container is labeled ' $\mathrm{O}$,' and the top of each successive layer is

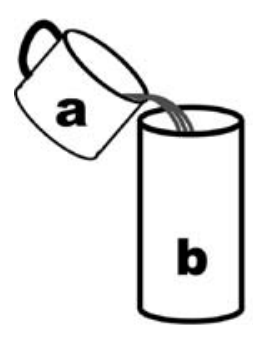

a

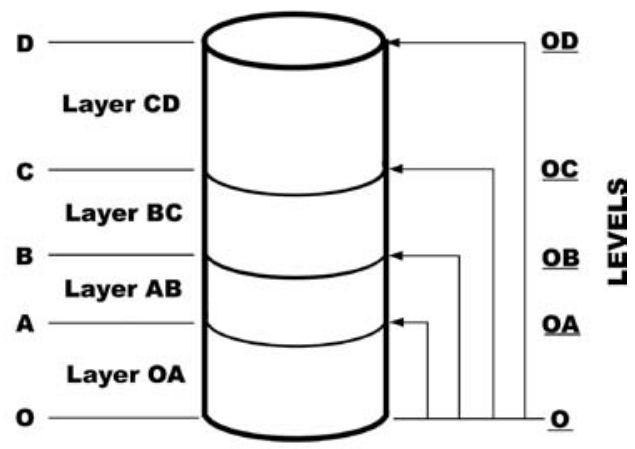

b

FIGURE 2 The embodied experience of handling liquids. Figure 2 a depicts a situation in which a distinct volume of water in cup " $a$ " is transferred into a larger cylindrical vessel "b". Figure $2 b$ illustrates the relationship between layers and nested levels. 
labeled 'A' 'B', and so on, the individual layers can be expressed as a pair of letters that denote their exact position in the stack: $\mathrm{OA}$ is the layer between the bottom $(\mathrm{O})$ and the top of layer $(\mathrm{A}), \mathrm{AB}$ is the layer between the top of layer $(\mathrm{A})$ and the top of layer (B), and so on. The layers describe distinct and discrete volumes of liquid in the container, and the relationships between these volumes can be expressed using bracket notation as ()()()$($ ). In contrast, the levels describe the volume of liquid from a specific point to the bottom of the container, encompassing all layers in between. The diagram illustrates another point: the levels in the container are nested, with level $\underline{\mathbf{O A}}$ included in level $\underline{\mathbf{O B}}$, which is included in $\underline{\mathrm{OC}}$, which is included in $\underline{\mathrm{OD}}$. As a result, removing the water (e.g., draining it, which is a way of inserting space) associated with any level (for example, $\underline{\mathbf{O B}}$ ) removes the water associated with all levels below that are included in that level. The arrangement of the levels within the container can be represented symbolically as the inequality

$\underline{\mathbf{O A}}<\underline{\mathrm{OB}}<\underline{\mathrm{OC}}<\underline{\mathrm{OD}}$, more generally as a nesting $(((())))$ ), or even more accurately as ()$)))$. These notations provide a simple means of describing the nesting of levels, which is explored in greater detail in the following sections.

\subsection{Abstract Target Domain: Comparison and Inequalities}

Not all notions are concrete. Consider the layers in Figure 3a, each of which has a different thickness. The layers define the corresponding levels and their relationship $\underline{\mathbf{O A}}<\underline{\mathbf{O B}}<\underline{\mathbf{O C}}<\underline{\mathbf{O D}}$. In the context of the water column, the
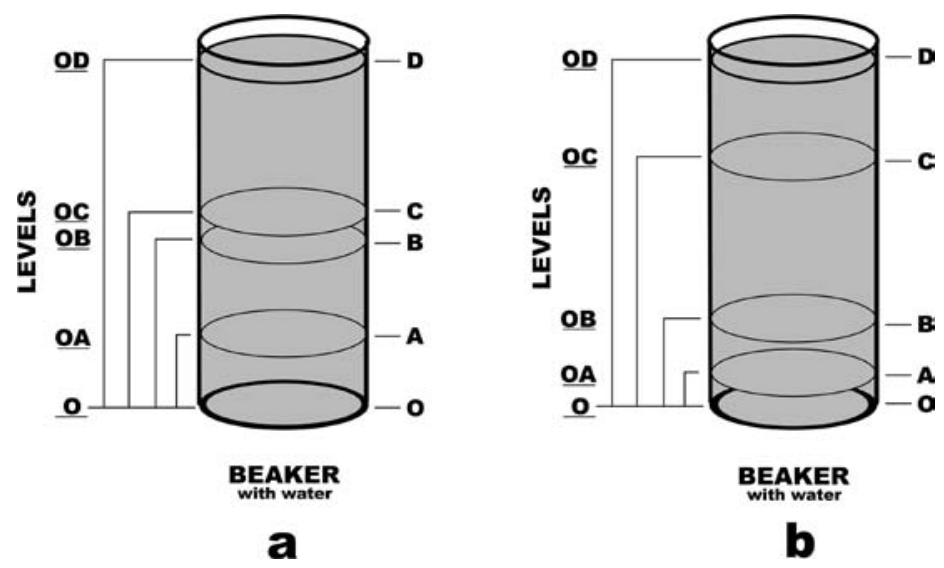

FIGURE 3 Inequalities. Figure $3 a$ and $3 b$ show two different arrangements of levels satisfying the same inequality. 
above inequality means that the volume with level $\underline{\mathbf{O A}}$ is included in volume $\underline{\mathrm{OB}}$, which is included in volume $\underline{\mathrm{OC}}$, which is included in $\underline{\mathrm{OD}}$.

Note that the levels in Figures $3 \mathrm{a}$ and $3 \mathrm{~b}$ can be represented by the same inequality, even though the levels in the two containers are different. In fact, an infinite number of combinations of levels fulfill this inequality. Therefore, the notion of levels, unlike that of layers, is abstract because levels are not dependent on space. In other words, levels represent a non-spatial ordering. Because of the absence of space, it is impossible to determine the extent to which any two levels differ. This independence from space is characteristic of intensity, magnitude, value and many other abstract concepts based on the SCALAR image schema. Additionally, the relationships among both the layers and the associated levels depend on the liquid-holding vessel; thus, the presence of the vessel must be taken into account, as expressed by the axiom below:

\section{Zero level $=$ empty vessel}

The next subsection discusses the difference between removing layers and removing levels. For the convenience of discussion, the three-dimensional rendering of water columns in all subsequent figures is replaced with diagrams of their cross-sections. Therefore, the issues will be considered only in terms of distance (i.e., the thickness of layers), eliminating any confusion between volumes, areas and lengths. The terms concatenation and addition will hereafter be used interchangeably.

\subsection{Layers are Distinct: Adding and Removing Layers}

To use level metaphors correctly, one must appreciate the structure of their source domain. This subsection discusses some source domain properties. As with all objects, layers are always discrete and distinct, and the sum of distinct layers is also distinct. Consider adding volumes of water to a cylindrical container. When they are concatenated, the volumes produce layers of greater thickness:

$$
\begin{aligned}
& \mathrm{OA}+\mathrm{AB}=\mathrm{OB} \\
& \mathrm{OA}+\mathrm{AB}+\mathrm{BC}=\mathrm{OC} \\
& \mathrm{OA}+\mathrm{AB}+\mathrm{BC}+\mathrm{CD}=\mathrm{OD} \\
& \mathrm{AB}+\mathrm{BC}=\mathrm{AC} \\
& \mathrm{BC}+\mathrm{CD}=\mathrm{BD} \\
& \mathrm{AB}+\mathrm{BC}+\mathrm{CD}=\mathrm{AD}
\end{aligned}
$$

Thus, one could say that layers exhibit an associative property. 


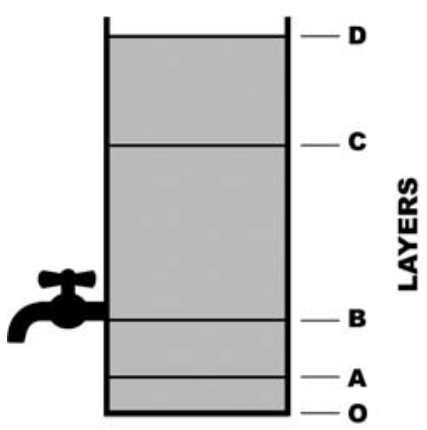

BEAKER

with water

a

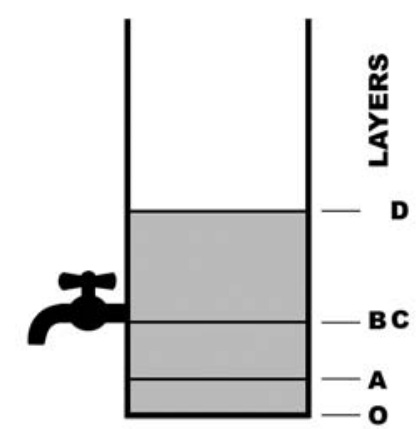

$(O A+A B+B C+C D)-B C$

b

FIGURE 4 Adding and removing layers. Removing layers is equivalent to removing parts of the whole.

If the layers are concatenated in a different order, they will still be part of the same column. For example, all layers can be arranged upside down. Therefore, layers also abide by a commutative property.

Pouring a volume of water into an empty vessel might change the shape of the volume but not the volume itself.

\section{Empty vessel $+\mathrm{OA}=\mathrm{OA}$}

Thus, layers also satisfy an identity property.

Layers can be not only concatenated but also removed from a column of water. Let us take away the middle layer $\mathrm{BC}$ by draining water from the vessel through the tap attached at the position indicated in Figure 4a.

Removing layers is, therefore, equivalent to removing parts of the whole; when one layer is taken out, all other layers remain in place (Figure $4 \mathrm{~b}$ ). However, this does not apply to levels.

\subsection{Combining Levels and Layers}

Layers and levels are closely related to one another. Without layers, one cannot express the notion of levels, and without levels, the metaphor MORE IS UP does not have any meaning. The current subsection examines this relationship in some detail. To differentiate between layers and levels, all level symbols are depicted with underlined bold letters. Let us add one layer at a time, starting from the bottom of the vessel (Figure $5 \mathrm{a}$ ). 


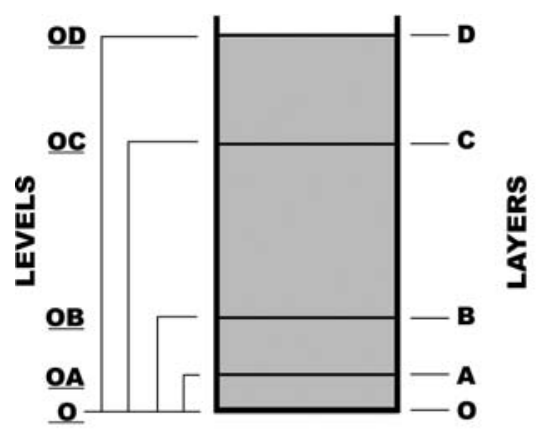

\section{$\underset{\text { with water }}{\text { BEAKER }}$}

a

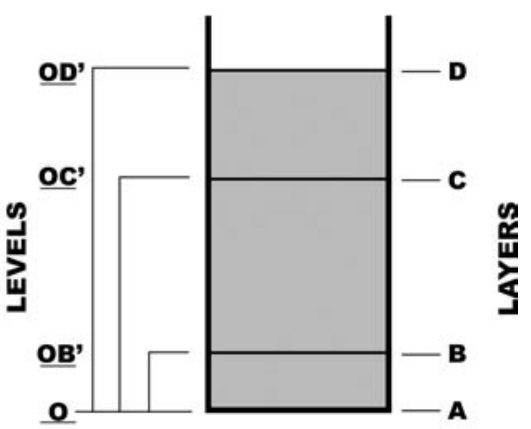

OD-OA $=A B+B C+C D$

FIGURE 5 Removal of the lowest level $\underline{\underline{O A}}$.

$$
\begin{aligned}
& \text { Empty vessel }+\mathrm{OA}=\mathrm{OA}=\underline{\mathrm{OA}} \\
& \mathrm{OA}+\mathrm{AB}=\mathrm{OB}=\underline{\mathrm{OB}} \\
& \mathrm{OA}+\mathrm{AB}+\mathrm{BC}=\mathrm{OC}=\underline{\mathrm{OC}} \\
& \mathrm{OA}+\mathrm{AB}+\mathrm{BC}+\mathrm{CD}=\underline{\mathrm{OD}}=\underline{\mathrm{OD}}
\end{aligned}
$$

The expressions above symbolically represent the essence of the metaphor MORE IS UP. As layers are added, the subsequent levels go up, resulting in the following inequality of levels:

$$
\underline{\mathrm{OD}}>\underline{\mathrm{OC}}>\underline{\mathrm{OB}}>\underline{\mathrm{OA}}>\underline{\mathbf{o}} \text {. }
$$

As outlined in Section 2.6, levels cannot be added, but they can be removed. Removing levels can result in removing one or more layers. This phenomenon is characteristic of all properties based on the SCALE image schema, including the notion of intensity. Figure 5 shows an example in which only one level has been removed.

$$
\underline{\mathbf{O D}}-\underline{\mathbf{O A}}=(\mathrm{OA}+\mathrm{AB}+\mathrm{BC}+\mathrm{CD})-(\mathrm{OA})=\mathrm{AB}+\mathrm{BC}+\mathrm{CD}
$$

Note that removing level $\underline{\mathbf{O B}}$ will result in two bottom levels being eliminated.

$$
\underline{\mathbf{O D}}-\underline{\mathbf{O B}}=(\mathrm{OA}+\mathrm{AB}+\mathrm{BC}+\mathrm{CD})-(\mathrm{OA}+\mathrm{AB})=\mathrm{BC}+\mathrm{CD}
$$


Furthermore, removing level $\underline{\mathrm{OC}}$ will result in three bottom levels being eliminated.

$$
\underline{\mathbf{O D}}-\underline{\mathbf{O C}}=(\mathrm{OA}+\mathrm{AB}+\mathrm{BC}+\mathrm{CD})-(\mathrm{OA}+\mathrm{AB}+\mathrm{BC})=\mathrm{CD}
$$

Finally, removing level OD will eliminate all four levels, leaving the vessel empty (Figure 6b).

$$
\underline{\mathbf{O D}}-\underline{\mathbf{O D}}=(\mathrm{OA}+\mathrm{AB}+\mathrm{BC}+\mathrm{CD})-(\mathrm{OA}+\mathrm{AB}+\mathrm{BD}+\mathrm{CD})=\text { empty vessel }
$$

This is an important observation. First, it means that the concept of the water column does not make sense without a vessel that contains liquid. Second, level "o" signifies the empty container. Third, zero does not stand for nothing; rather, it represents the absence of content in a vessel that is very much concrete and distinct. This situation is not unique to containers with liquids. For example, it is impossible to detect blackness (i.e., the zero level of light intensity) without a light sensor; thus, black is not a color as such but, instead, the absence of light, which is typically expressed with paint that has been formulated to absorb light (and that is generally described as "black paint"). Similarly, to gauge no voltage (i.e., the zero level of voltage), one must use a voltmeter; to make no profit (i.e., the zero level of profit), one must have a business; and to pay no taxes when others do still requires a tax collector.

In summary, the notions of levels and layers represent two distinct but related ways of thinking. Layers fit the part-whole relationship associated with a

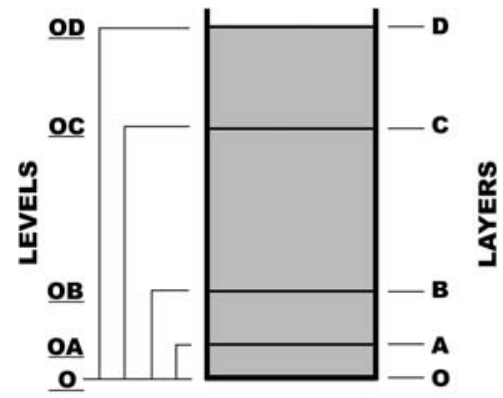

BEAKER

a

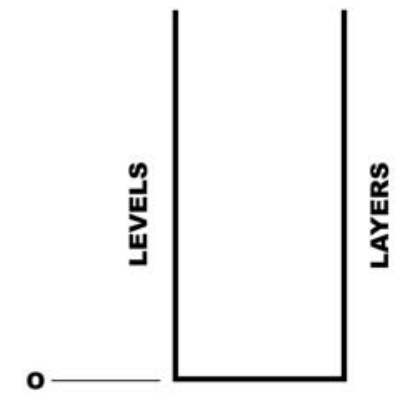

OD-OD = empty vessel

b

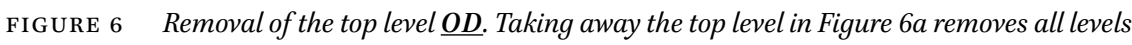
included in it and leaves the container empty. The empty container in Figure $6 b$ represents level zero. 
single scale, whereas levels behave as nested scales, as described by the SCALE image schema. The issue of scales is discussed further in the section about cognitive products.

\subsection{Making Communication Precise: Identical Layers}

Previously, the layers that constitute the column of water were assumed to be of different thickness, indicating that an infinite number of layer combinations can satisfy the same inequality of levels. This framework presents communication problems because each listener will come up with their own arrangement. Using layers of identical thickness makes the relationship between levels independent of the layer thickness. Imagine a column that is created by pouring water into a cylindrical vessel with the same cup. Because all layers are identical, the difference between subsequent levels is fixed and equal to one layer (thickness " $h$ ", in this case), which imposes on the concept of levels a sense of regularity that is associated with layer repetition (Figure 7).

Levels can now be expressed in terms of the layer thickness:

$$
\begin{aligned}
& \underline{\mathbf{O A}}=\mathrm{OA}=\mathrm{h} \\
& \underline{\mathbf{O B}}=\mathrm{OA}+\mathrm{AB}=\mathrm{OB}=2 \mathrm{~h} \\
& \underline{\mathbf{O C}}=\mathrm{OA}+\mathrm{AB}+\mathrm{BC}=\mathrm{OC}=3 \mathrm{~h} \\
& \underline{\mathbf{O D}}=\mathrm{OA}+\mathrm{AB}+\mathrm{BC}+\mathrm{CD}=\mathrm{OD}=\mathrm{h}+\mathrm{h}+\mathrm{h}+\mathrm{h}=4 \mathrm{~h}
\end{aligned}
$$

Thus, taking away one layer (it does not matter which one) will produce a column of water of the same thickness.

$$
\begin{aligned}
& \mathrm{OA}+\mathrm{AB}+\mathrm{BC}+\mathrm{CD}-\mathrm{OA}=3 \mathrm{~h} \\
& \mathrm{OA}+\mathrm{AB}+\mathrm{BC}+\mathrm{CD}-\mathrm{AB}=3 \mathrm{~h}
\end{aligned}
$$

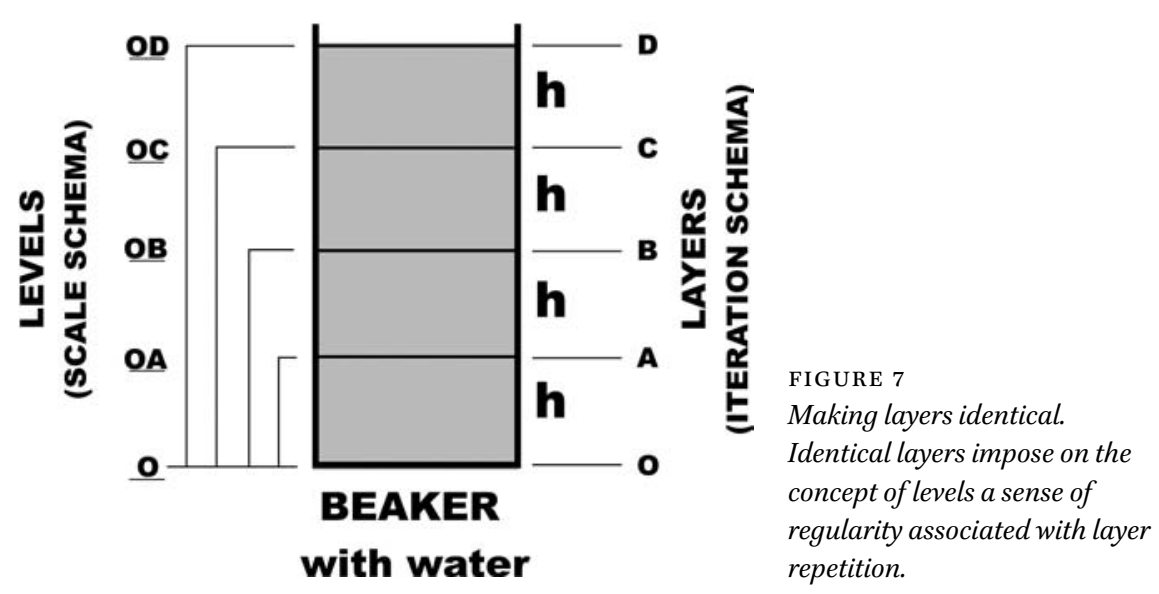




$$
\begin{aligned}
& \mathrm{OA}+\mathrm{AB}+\mathrm{BC}+\mathrm{CD}-\mathrm{BC}=3 \mathrm{~h} \\
& \mathrm{OA}+\mathrm{AB}+\mathrm{BC}+\mathrm{CD}-\mathrm{CD}=3 \mathrm{~h}
\end{aligned}
$$

Note that taking away a level removes all levels that are included in that level.

$$
\begin{aligned}
& \underline{\mathbf{O D}}-\underline{\mathbf{O A}}=(\mathrm{OA}+\mathrm{AB}+\mathrm{BC}+\mathrm{CD})-(\mathrm{OA})=\mathrm{h}+\mathrm{h}+\mathrm{h}=3 \mathrm{~h} \\
& \underline{\mathbf{O D}}-\underline{\mathbf{O B}}=(\mathrm{OA}+\mathrm{AB}+\mathrm{BC}+\mathrm{CD})-(\mathrm{OA}+\mathrm{AB})=\mathrm{h}+\mathrm{h}=2 \mathrm{~h} \\
& \underline{\mathbf{O D}}-\underline{\mathbf{O C}}=(\mathrm{OA}+\mathrm{AB}+\mathrm{BC}+\mathrm{CD})-(\mathrm{OA}+\mathrm{AB}+\mathrm{BC})=\mathrm{h} \\
& \underline{\mathbf{O D}}-\underline{\mathbf{O D}}=(\mathrm{OA}+\mathrm{AB}+\mathrm{BC}+\mathrm{CD})-(\mathrm{OA}+\mathrm{AB}+\mathrm{BC}+\mathrm{CD})=\text { empty vessel }
\end{aligned}
$$

These and other properties of levels are not affected by the layer thickness.

Please note that the act of filling the container with identical volumes of water is an example of the ITERATION image schema (Johnson, 1987: 126) which unfortunately has never been defined by Johnson. The name of the schema suggests the act of repeating a unit. Some possible examples include such units as steps in the case of walking, notes in the case of sound, lengths of measure tapes, degrees of a thermometer, etc. But they also include the repetition of layers in the case of containers, and containers in the case of their collections. For the unit to be repeated it must be distinct and discrete, meaning it must be possible to separate it from other units with space. All units can potentially be repeated without an end. The experience of adding identical layers can therefore be reasoned in terms of the ITERATION image schema, the same way as the experience of levels can be understood in terms of the SCALE image schema (Figure 7). They represent two distinct ways of thinking which could be schematically represented with nested brackets as $((((\ldots))))$ in the case of levels and as concatenated brackets ()()()()$\ldots$ in the case of layers. As a result, the interaction between levels and layers can be examined by considering the interaction between the SCALE and ITERATION schemas which is discussed in the next section.

\subsection{Conceptual Duality: Expressing Abstract Notions Using Concrete Ones}

There are many similarities between the approach outlined in this paper and the account of the MORE IS UP metaphor presented by Kövecses. Both frameworks suggest an interaction between two experiential concepts: "When we pour water into a glass or when we add more of something to a pile, we bring together two distant conceptual domains (i.e., quantity and verticality) in a single domain, in which the two can be found simultaneously" (Kövecses, 2010: 187). The process of "bringing up two distant conceptual domains" is defined in this paper as conflating levels with layers, which is briefly described below. 


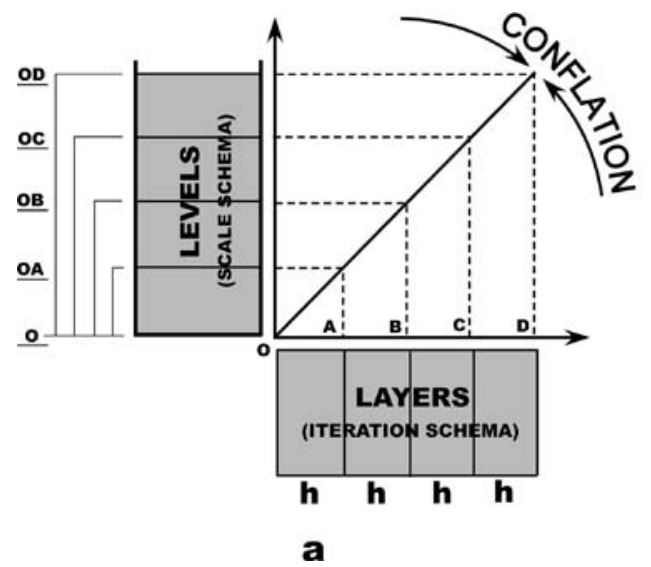

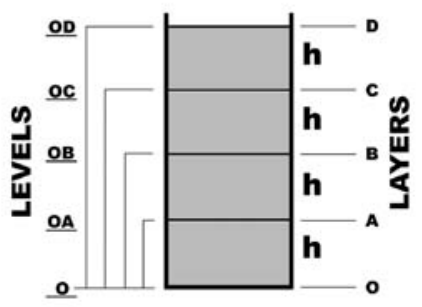

COLUMN

FIGURE 8 Conflating the SCALE schema (levels) with the ITERATION schema (layers). All columns of liquid can be understood as repetition of discrete layers or as nested levels, or both.

When all layers in a column are identical, they impose the regularity of their repetition onto nested levels and reduce the infinite number of possible level combinations satisfying the inequality $\underline{\mathbf{O}}<\underline{\mathbf{O A}}<\underline{\mathbf{O B}}<\underline{\mathbf{O C}}<\underline{\mathrm{OD}}$ to a single arrangement that is independent of the layer thickness. This in turn allows the two distinct concepts (Figure 8a) to be conflated into a single notion of a column (Figure 8b), which is understood here as a dimension.

In this context, conflation means that the idea of a water column can be considered in terms of the repetition of discrete layers (an action and partwhole relationship) or as nested levels (states and inequalities). Additionally, the two ways of thinking modify each other: the layers will impose a sense of regularity and dynamics onto the levels and reduce their infinite variation to one arrangement, and the nested nature of levels will impress a sense of confinement, order and discreteness of the state onto a potentially never-ending process of repeating layers. This conceptual duality extends to other aspects of conflation. For example, in the context of repetition, the notion of continuity can be understood as the absence of space between layers. The column in Figure $8 \mathrm{~b}$ is continuous because there is no gap between discrete layers, and the layers can always be separated by inserting space between them. However, the notion of nested levels defines continuity in terms of the indivisibility of units. These levels are continuous because it is not possible to divide the units without destroying their nested arrangement.

The concept of conflation that combines levels with layers demonstrates the inner working of metaphors: the abstract (non-spatial) sense of intensity and its levels can be understood and expressed in terms of concrete 
(space-based) layers. For this to occur, the two domains must share a common structure, at least in part; thus, readers must be able to think about an abstract concept in the way they think about columns of water.

\subsection{The Idempotence of Scalar Addition}

The concept of idempotence is significant for linguistics because it explains the role of adjectives and adverbs in language. Consider the phrase "a red apple." This phrase is likely to be an abbreviation of a more complete expression: "the average-red surface of an apple", where the modifier average specifies the intensity of redness, and the word apple indicates the extent of the property (in this case, area). This issue is elaborated below in terms of levels and layers.

As demonstrated in Figures 3 and 5, and stated by their arguments, layers are distinct and discrete volumes that can be added and subtracted with ease. However, this characteristic does not apply to levels, which cannot be added because they are not distinct. Adding a volume of 50 degree Celsius water to another volume of water of the same temperature will change the volume but not the temperature. Adding red paint of some intensity to red paint of identical intensity will not change the intensity level of the red paint, but it will change its area or thickness. Similarly, adding a volume of water of some level to a volume of the same level will increase the volume of water but not its level (Figure 9).

This behavior might seem counterintuitive in the context of filling up the beaker shown in Figure 2, where adding layers changes levels. The problem

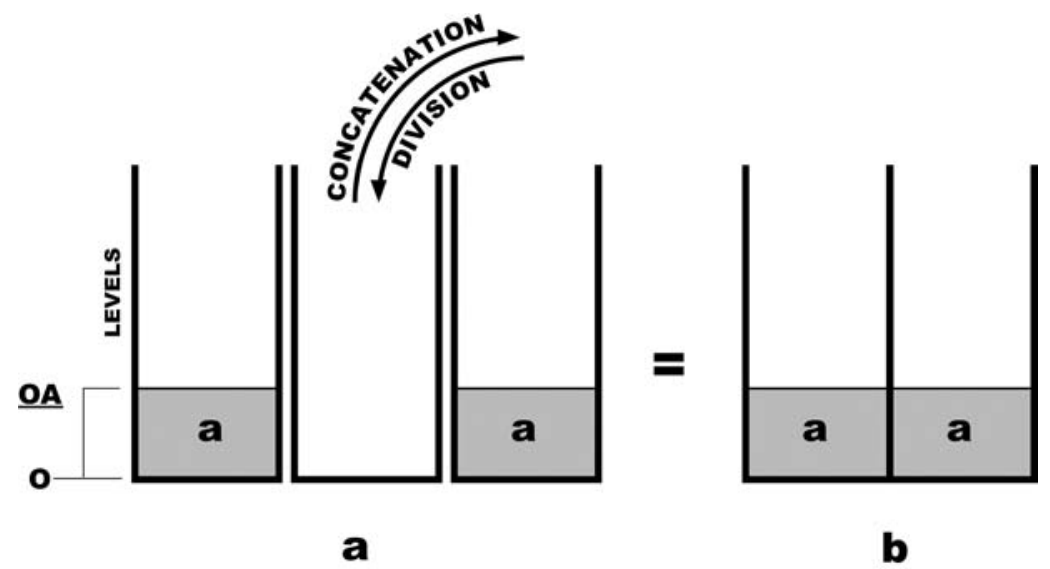

FIGURE 9 The idempotence of scalar addition. The diagrams show that one can add discrete entities only if they share a property of the same level. Adding involves removing space from between units and dividing involves inserting space between them. 
arises whenever adding levels is confused with adding layers. The diagram in Figure 9 shows the conditions under which addition can take place. Recall the temperature and intensity levels from the examples at the beginning of this section; it is the volume or area that changes, not the level of paint intensity or the water temperature. In fact, one can add discrete entities only if they share a property of the same level, such as in terms of intensity, value or degree. If the temperatures of the added water, the intensity of the added paint, or the concentrations of drinks differ, we are not addressing a process of addition but a process of mixing, which is more complex than addition. Adding entities of the same level (e.g., of intensity/value/temperature) is an idempotent process that is depicted symbolically below:

$$
\begin{aligned}
& \text { Empty container }+ \text { empty container }=\text { empty container } \\
& \underline{\mathrm{OA}}+\underline{\mathrm{OA}}=\underline{\mathrm{OA}} \\
& \underline{\mathrm{OB}}+\underline{\mathrm{OB}}=\underline{\mathrm{OB}} \\
& \underline{\mathrm{OD}}+\underline{\mathrm{OC}}=\underline{\mathrm{OC}}=\underline{\mathrm{OD}}
\end{aligned}
$$

Adding levels (for example, $\underline{\mathbf{O A}}+\underline{\mathbf{O B}}$ ) does not make sense because by definition $\underline{\mathbf{O A}}$ is already included in level $\underline{\mathbf{O B}}$.

The common assumption that entities of the same type can always be added ignores the fact that all entities are described by the levels of their properties. Therefore, one cannot express properties without simultaneously specifying their intensities and the extents of those intensities. For example, one cannot paint an area without a color of some intensity (e.g., bright red vs. dull red) and an extent (i.e., area). Whenever the intensity is not stated, humans tend to assume the average or most salient level of the intensity. These issues will become clear when they are considered in the context of cognitive products. ${ }^{1}$

The notion of addition also requires some clarification. If one considers containers as an example, then addition can be conceptualized as removing space from between identical beakers that share the same level of liquid (Figure gb) or, more accurately, as removing all empty vessels between the addends. As mentioned above, this process is called concatenation. Division can then be thought of as a procedure that is opposite to concatenation because it involves inserting space (i.e., an empty vessel or vessels) between beakers filled to the same level. Concatenation and division involve thinking in terms

1 The addends are two products sharing an identical intensity of a property: \{Product + Product $2=\left(\left(\right.\right.$ intensity $_{\mathrm{OA}} \cdot$ extent $\left._{1}\right)+\left(\right.$ intensity $_{\mathrm{OA}} \cdot$ extent $\left.\left._{2}\right)\right)=\left(\right.$ intensity $_{\mathrm{OA}}\left(\right.$ extent $_{1}+$ extent $\left.\left.\left._{2}\right)\right)\right\}_{\text {property A. }}$. 
of a part-whole relationship, which involves only changes of extent (here, the number of concatenated vessels). Please note that concatenation and division can be used only in relation to multiples of discrete and distinct entities, such as layers and containers, but not levels. To be discrete and distinct means that such entities are or could be spatially separated from similar entities. This procedure cannot be applied to levels that, by definition, do not involve space. As a result, levels can only be differentiated and never divided or concatenated; this is the subject of the next section. Evidence that humans differentiate levels can be found in the way that we talk about them. All properties that can be expressed using levels tend to involve some form of differentiation as a means of creating new levels. For example, properties such as poverty, leadership, impairment, cognitive demand, and stress can all be a part of the phrase "differentiating levels ...." However, whenever division is used in the context of levels, it tends to involve segregating or sorting vessels into groups or sets of identical levels rather than introducing new levels. The sentence, "To make it easier, let's divide the levels of intensity into three types" (Swanson, 2009), can be considered an example of such a usage. This issue, which is discussed in the next subsection, is essential for understanding how cognitive products (discussed in Subsection 2.8) are used in linguistics.

\subsection{Differentiating Levels}

Unlike the operation of division, which involves the insertion of space, differentiation is entirely a mental process. Imagine an open but opaque beaker filled with a non-transparent liquid (e.g., Figure 10z). The only things that one can see in this case is the exterior of the beaker and the top surface of the liquid (meniscus), which is the upper-most level of the liquid column. One could say that both the beaker and the meniscus are public because they can be observed by any number of individuals, which is possible only if such entities exist in the space among the viewers. Therefore, levels other than the top one are private because they cannot be directly experienced by anyone other than their subject. Such levels are invoked separately by each individual engaged in the experience. To make them known to other observers, these private experiences must be expressed publically, which is usually achieved by marking layers on the outside surface of the container. Subsequently, the layers become the public representation of the private notion of levels. One could also say that, as a relationship, the idea of levels exists at the small scale of the individual (in the mind), while layers occupy the large-scale space that exists among the individuals. However, when they are considered in the context of containers, levels correspond to the small scale of content, and the containers represent the larger scale in which more than one layer and container can exist. 
Furthermore, nested levels signify states (i.e., a static relationship) because no level can be conceived of without all lower levels existing simultaneously. In contrast, layers represent the potentially never-ending process of repetition.

With this interpretation in place, let us more closely consider the notion of differentiation by referring to Figure 10. Although levels cannot be divided or concatenated, they can always be differentiated by the observer.

First, consider the column of liquid in Figure 10a, which combines layer o-1 with level o1. One can differentiate any number of levels within the column. Figure 10 b shows levels $\mathbf{1} / \mathbf{2}$ and $\mathbf{2} / \mathbf{2}$, which result in two layers- $0-\mathbf{1}$ and $\mathbf{1 - 2 -}$ of identical thickness. Figure 1oc depicts four levels $1 / 4,2 / 4,3 / 4$ and $4 / 4$, which give rise to four identical layers $0-1,1-2,2-3$, and $3-4$. In fact, one can conjure an infinite number of sublevels within column 01, as depicted in Figure 10z, which presents a rectangle whose area is black due to the infinite number of level marks. Please note that the levels expressed by the fractions are nested. For example, the levels $1 / 4,2 / 4,3 / 4$ and $4 / 4$ in Figure $10 c$ represent a nesting in which level $4 / 4$ contains level $3 / 4$, which contains level $2 / 4$, which contains level $\mathbf{1} / \mathbf{4}$, which contains level zero, which represents an empty vessel. Such

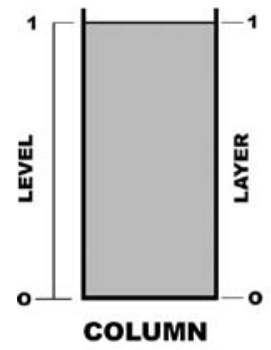

a

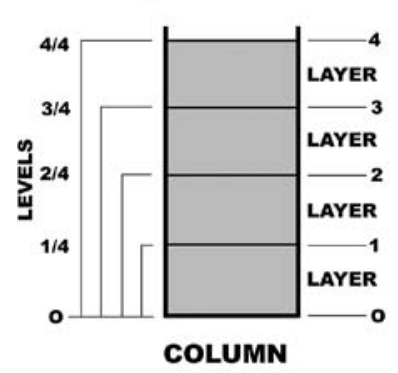

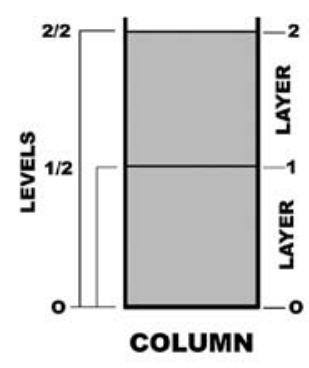

b

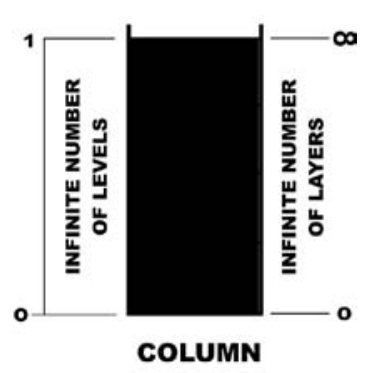

$\mathbf{z}$

FIGURE 10 Differentiation. One can differentiate an infinite number of levels within any container with liquid. 
nesting is typically expressed as an inequality: $4 / 4>3 / 4>2 / 4>1 / 4>0$. Because the difference between successive levels is constant and equal to the thickness of the common layer, the levels can be expressed as the sums of layers $1 / 4+1 / 4+$ $1 / 4+1 / 4$ for $4 / 4,1 / 4+1 / 4+1 / 4$ for $3 / 4,1 / 4+1 / 4$ for $2 / 4$, and $1 / 4$ for $1 / 4$; this characteristic makes discussing columns in terms of a part-whole relationship possible. Figure $10 \mathrm{z}$ highlights the differences in the human interpretation of layers and levels. Because the act of differentiating occurs in the observer's mind, the conceived levels must be expressed publically to be appreciated by others, such as by making marks on the vessel wall. Because the difference between the levels could be zero, there is no limit to how many levels one can invoke. However, this way of thinking cannot be applied to layers that by definition are discrete and concrete; layers of zero thickness are no longer distinct, and more importantly, adding layers of no thickness would not recreate the column of which they are a part.

As mentioned above, differentiating additional levels results in an equal number of identical layers. For example, differentiating three additional levels in Figure 11 bives rise to three layers, each of which has a thickness equal to one third of the original layer in Figure 11a.

To preserve the volume while differentiating three additional levels, the single beaker of a rectangular base (Figure 11a) requires three smaller beakers (Figure 11b) with bases equal to one third of that of the original vessel. ${ }^{2}$ This case is similar to differentiating millimeters within the centimeters of a ruler; to preserve the extent of one centimeter when differentiating millimeters, the space between two subsequent millimeter marks must be one tenth of the

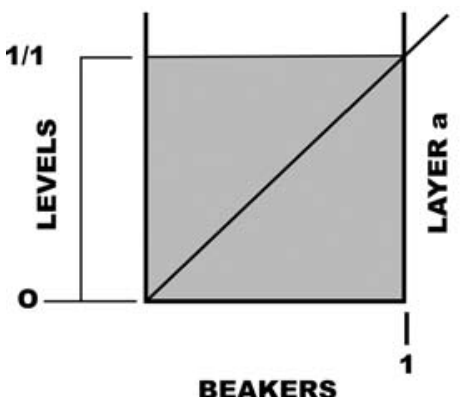

a

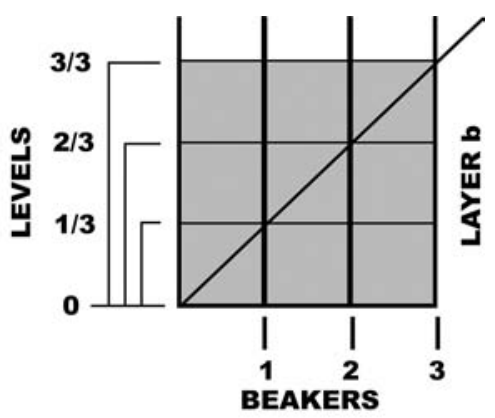

b

FIGURE 11 Differentiating levels within the unit. Figure na depicts a beaker with one level. Figure $n$ b shows how differentiating new levels affects layers and containers.

2 The arrangement is significant for cognitive products that associate containers with levels. 
centimeter. In the case of columns, this preservation is ensured by the $45^{-}$-degree diagonal lines in Figure 11 that define the bases of all beakers. Please note that the intersection of levels and the beaker walls always creates squares, irrespective of how many sublevels are differentiated. The conclusions drawn based on these squares are also applicable to cubes. All these phenomena are significant for the use of cognitive products in linguistics, as discussed in the next two subsections, and for understanding the various ways in which products can be represented, which is discussed in Subsection 2.10.

\subsection{Cognitive Products in the Context of a Liquid Column}

The notion of cognitive products is probably the most important idea presented in this paper because, as I argue, most if not all linguistic expressions are cognitive products in one way or another. I would like to draw the reader's attention to the convention used in this paper: the first factor of any product always represents the level, and the second factor, which is understood as an extent of the product, represents the number of units of some type. Additionally, it should be noted that numbers are used in this paper for the convenience of written communication.

As argued in the previous subsections, the columns of liquid are conflations of two distinct ways of thinking: one involving nested levels and the other, concatenated layers. Consider the three concatenated beakers in Figure 12a, each of which contains four identical levels of liquid.

The product is the totality of all unique associations (marked with large dots in Figure 12) between the containers and the levels of their contents.

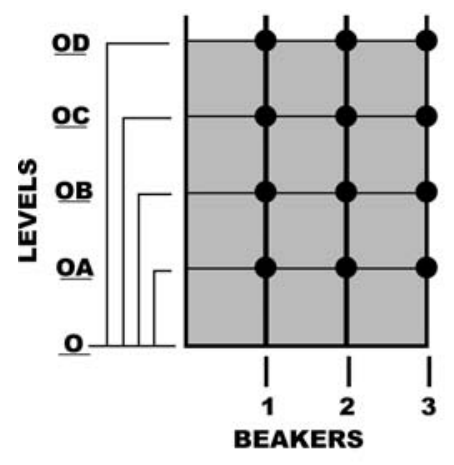

a

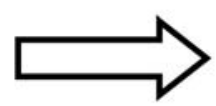

FIGURE 12 Cognitive products in the context of a liquid column. Product is the totality of associations between levels and containers depicted with large dots (Figure 12a). The same product could be expressed using layers and beakers (Figure 12b). 


$$
\begin{aligned}
\text { Product } & =(\text { levels within the unit }) \cdot(\text { unit repetition }) \\
& =(4 \text { levels }) \cdot(3 \text { containers })=12 \text { associations }
\end{aligned}
$$

Because the difference between the levels is perceived as being constant $(\underline{\mathbf{O D}}-$ $\underline{\mathbf{O C}}=\underline{\mathbf{O C}}-\underline{\mathbf{O B}}=\underline{\mathbf{O B}}-\underline{\mathbf{O A}}=\mathrm{a}=\mathrm{const}$ ), the column can be interpreted in terms of layers rather than levels, which makes the task of thinking about products and expressing such thoughts much easier.

$$
\begin{aligned}
\text { Product } & =(4 \text { levels }) \cdot(3 \text { containers })=(4 \text { layers }) \cdot(3 \text { containers }) \\
& =12 \text { associations }
\end{aligned}
$$

As a result, a column can be viewed as a sum of layers, and a product defined in this way can be understood as the relationship between entities of the same scale (Figure 12b). Such an interpretation encourages thinking about multiplication in terms of, for example, addition, part-whole relationships, and scalefree reality. This approach makes life easier. However, in the process, important insights are inadvertently lost: that cognitive products associate the idea of unit repetition (here, beakers) with nested levels, that such products merge the notions that exist at two different scales, that they combine private ideas with public expression, states with their repetition, and levels with their extent, to mention only a few of the salient issues. Thus, the idea of columns cannot be fully understood without the notion of a container (large scale) and the levels of its content (small scale), without internal and private experiences and their public articulation, without private observers and public expressions, and so on.

Please also note that products cannot be formed without vessels of some type, although they can exist without content. Empty vessels can be multiplied (repeated) as easily as vessels that hold contents. The product constructed of empty vessels (regardless of how many) is always zero (in other words, an empty but larger vessel), which follows directly from the property of idempotence. Although multiplying by a fraction or by zero is perfectly logical in mathematics, from the cognitive product perspective, multiplying zero times (in the sense of repeating) does not make sense. One can repeat something one, two, three, or infinitely many times but not "zero times," which simply means not multiplying at all. Similarly, multiplying something "a fraction of times" is meaningless in the context of units (here, containers) as this would imply partitioning the units, which are, by definition, indivisible. Because units can be repeated only as intact wholes, the number of repetitions must be a natural number. Consider a single-level container as an example. Multiplying it by 0.5 would mean dividing the container in half. However, due to the flexibility of 
the product concept, one can differentiate another level within the unit and multiply the unit of 1/2-level once. Alternatively, one can differentiate nine additional levels within the unit and repeat the unit of 1/10-level five times, and so on. This and related issues are elaborated in Section 2.10. In the next section, I discuss a small selection of linguistic examples in the context of cognitive products.

\subsection{The Use of Cognitive Products in Language}

The analysis in the preceding subsections should help the reader recognize cognitive products in linguistic expressions. Due to the constraints of the article, this study is limited to a few simple examples. In essence, linguistic products are phrases that specify intensities and their extents for the various properties attributed to an object or the subject of the expression:

$($ PRODUCT $=($ smaller-scale unit $\odot$ larger-scale extent of unit repetition) $\left.)_{\text {property }}\right)_{\text {object/subject }}{ }^{3}$

Consider the phrase, "the jar of bright-red paint," which combines the intensive factor (bright red) with the extent of the intensity (the paint volume defined by the jar) of the property (the color of the paint).

$\left(\text { PRODUCT }=(\text { bright red } \odot \text { volume })_{\text {red color of the paint in }}\right)_{\text {a jar }}$

For comparison, consider the phrase "the bright-red jar with paint," which could be represented as

$\left(\text { PRODUCT }=(\text { bright red } \odot \text { area })_{\text {red color of }}\right)_{\text {the jar }}$

Now consider the sentence, "France has a high level of public debt," which combines the intensive factor (high level) with the extent of one as the debt is related to a single country.

$\left(\text { PRODUCT }=(\text { high level } \odot 1)_{\text {public debt }}\right)_{\text {France }}$

Cognitive products can also be identified in more complex sentences, such as, "I worked very hard for thirty days on my paper."

$\left(\text { PRODUCT }=\left(\left((\text { very hard } \odot \text { thirty })_{\text {effort }}\right)_{\text {days }}\right)_{\text {my paper }}\right)_{\text {pronoun "I" }}$

3 The symbol $\odot$ represents multiplication in the context of linguistic phrases. 
Cognitive products can also be used to express a change in a sentence, such as "The snow melted completely overnight." This sentence uses three products:

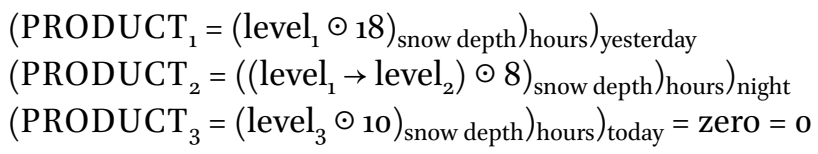

Note that the hours used in the example are arbitrary. The word "completely" suggests no presence of snow, indicating that level ${ }_{2}=\operatorname{level}_{3}=0$, and Product ${ }_{3}$ equals zero. ${ }^{4}$ Also note that Product ${ }_{2}$ involves a change of levels, which assumes a steady melting of snow as the most salient form of the change. The process of changing depth is not a product but an infinite series of sub-products of decreasing levels (a sloping line), which can be converted into a single product (a rectangle with 8 hours as a base) by differentiating a 1/2 sublevel within Level.

$$
\left(\text { PRODUCT }_{2}=\left(\left(\left(\text { level }_{1 / 2}\right) \odot 8\right)_{\text {snow depth }}\right)_{\text {hours }}\right)_{\text {night }}
$$

The concept of a cognitive product explains the flexibility of many linguistic expressions. Consider the sentence, "John has 20 dollars," which could mean that he has one $\$ 20$ bill, two $\$ 10$ bills, four $\$ 5$ bills, twenty $\$ 1$ coins, and so on. Considering this concept leads to the issue of the various ways in which products can be represented. For convenience, I will use numbers rather than linguistic expressions to elaborate on this topic.

\subsection{Ways of Representing Cognitive Products}

Cognitive products provide a flexible mode of reasoning. Consider the four volumes of liquid in Figure 13 as an example. Irrespective of the arrangement, all three figures represent the same product.

$$
\begin{aligned}
\text { Product } & =(4 \text { levels }) \cdot(1 \text { beaker })=(1 \text { level }) \cdot(4 \text { beakers }) \\
& =(2 \text { levels }) \cdot(2 \text { beakers })=4
\end{aligned}
$$

Thus, one can think about the products in at least three distinct ways: as a single container (a unit) with the nested content (intensive representation in Figure 13a), as a collection of containers with the content having only one level (extensive representation in Figure $13 \mathrm{~b}$ ), and as a collection in which the numbers of containers and levels is the same (square representation in Figure 13c). These modes of reasoning are discussed below in some detail.

4 Compare this sentence with the statement "Most of the snow melted overnight". 


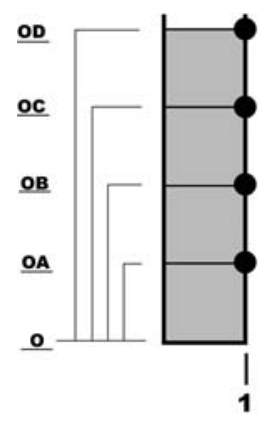

a

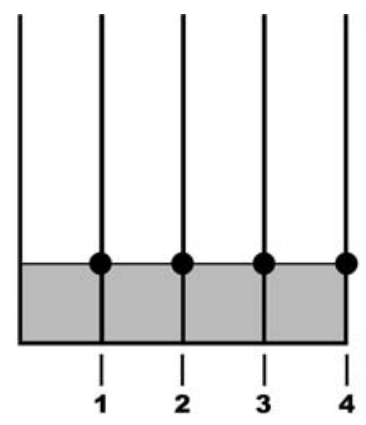

b

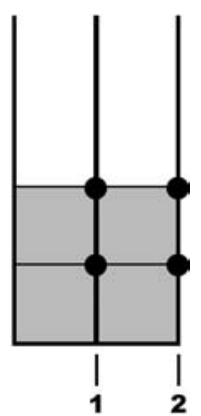

c

FIGURE 13 Ways of representing cognitive products. Figure 13 a shows an intensive representation; Figure $13 b$ an extensive representation; and Figure $13 \mathrm{c} \mathrm{a}$ square representation.

The extensive representation of a product involves multiples of the unit with one level only ( 1 level $\cdot 4$ beakers in Figure $13 \mathrm{~b}$ ), which focuses attention on the extent, single scale and part-whole relationship [( 1 level $\cdot 1$ beakers $)_{\text {unit }}+$ ( 1 level $\cdot 1$ beakers $\left.)_{\text {unit }}+(1 \text { level } \cdot 1 \text { beakers })_{\text {unit }}+(1 \text { level } \cdot 1 \text { beakers })_{\text {unit }}\right]$. Manipulating such products requires less effort than simultaneously handling multiple levels and their extents. Reducing products to one scale makes them public (i.e., the single level and single layer are then interchangeable), which facilitates their offloading into the environment in which they are expressed, stored, shared and manipulated publically (e.g., added/subtracted, linked/associated, or arranged into sets or chains) as distinct objects. Unfortunately, manipulating extents alone encourages algorithmic thinking (i.e., reasoning in terms of rules and procedures), which promotes learning by rote. Thinking in terms of one scale also encourages the view of products in terms of a partwhole relationship. From this perspective, multiplication is seen as nothing more than a convenient method of adding. ${ }^{5}$

The intensive representation of a product, however, involves a single indivisible unit (e.g., beaker) that contains all levels of the product (4 levels . 1 beaker in Figure 13a). This mode is used in various types of comparisons. The easiest way to compare two different products of some property is by converting them into their respective intensive representations. For example, by utilizing two identical containers, one can compare two volumes exclusively in terms of their heights, which make the process easier and, hence, more reliable. The extent of all intensive representations is 1 , which focuses the attention on levels. Intensive representations can be used to create larger products

5 The issue is discussed by Keith Devlin (2008). 
by replicating the unit [e.g., ( 4 levels $\cdot 1$ beaker $)_{\text {unit }} \cdot 3$ ]. The result of repeating such a unit is always a rectangular product, as discussed below.

Rectangular representation involves a product in which the repetition of the units and the number of levels within it are different and greater than one. Note that collections of units of different levels are not cognitive products. Variable levels represent cognitive structures that are more difficult to manage than the steady-state change represented by products, which is why the variations are often converted into products. The transformation of a dynamic change into an equivalent product is outlined in the comments about the sentence "The snow melted completely overnight" in the previous section.

The square representation of a product has the same number of levels as vessels. The example in Figure 13c shows two containers with two levels each, which results in product $2_{\text {levels }} \cdot 2_{\text {vessels }}=4$. When it is expressed, the product becomes squares capable of filling space without any gap. For this reason, square representation is used to compare the areas of irregular figures. This procedure is called squaring (Hobson, 1913) and is the method of constructing a square product with an area that is identical to that being measured. This process involves differentiating increasingly smaller square units until the whole area is completely covered in a finite number of steps. Such differentiation is an easy task for some products (e.g., 4, 9, and 16) but not for others (e.g., 2, 3, 5, 6, and 7). To illustrate this concept, consider 3 as a product. There is no problem with its intensive (Figure 14a) and extensive (Figure 14b) representations. However, the square representation is challenging because the layers cannot form a square (Figure 14c). The only way to overcome this situation is by differentiating increasingly smaller square units (refer to Section 2.7) until the irregular shape in Figure $14 \mathrm{C}$ is converted into the square shown in Figure 14d. This process is typically referred to as finding the square root of a product.

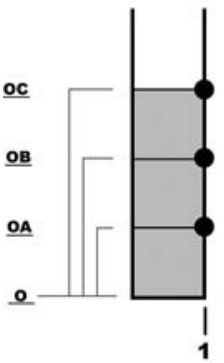

a

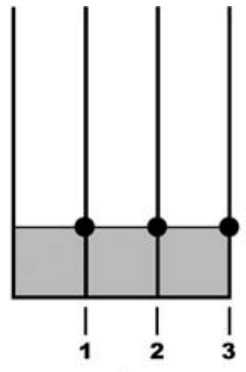

b
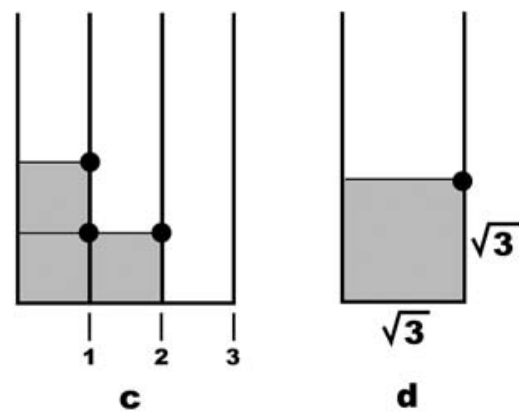

d

FIGURE 14 Square representation of product3. The square representations of some products are not well-defined. 
In the case of 3 , it is not possible to find the square root because $\sqrt{3}$ (the number of unit repetitions) cannot be converted to a natural number, irrespective of how many new levels are differentiated within the original unit. In other words, the square root of 3 is an irrational number, which cannot be expressed as a fraction that as described in the section about cognitive products, can always be converted into a natural number of repetitions. Therefore, the square representation of 3 can only be approximated.

$$
\text { Product }=(\sqrt{3})_{\text {levels within unit }} \cdot(\sqrt{3})_{\text {repetition }} \cong 1.73 \cdot 1.73=0.0173 \cdot 173=2.9929
$$

The product of the approximated factors is less than 3 , and adding more decimal places will not change this.

The argument above suggests that the notion of products can be used for measurements that can be interpreted in the context of cognitive linguistics to improve the precision of communication. Measuring a concrete property, such as length, area or mass, involves expressing the property as multiples of its respective concrete units. For example, areas can be represented by multiples of units based on area (concrete $\rightarrow$ concrete mapping). However, measuring private and abstract properties (e.g., the intensity of pain or level of knowledge) can be achieved only by expressing those properties as multiples of concrete and public units (abstract/private $\rightarrow$ concrete/public mapping). For example, the intensity of pain can be presented publically by using any one of a few pain-range scales that are essentially short lines divided into equal sections (Williamson and Hoggart, 2005). Therefore, such measurements are a form of the conceptual metaphor in which the concrete domain is mapped onto the abstract domain. Note that describing a sensation as "very painful" is not as accurate as reporting that the level of pain is seven on a scale of ten. Furthermore, reports that use scales allow rates of change and many other issues to be discussed (Maranell, 2009). This sense of measurement is as much a part of linguistics as it is of science.

\section{3}

\section{Conclusions}

Handling water and other liquids provides a rich and productive structure of embodied experiences that can be used as a source for a target domain of interest. For the experience of a water column to serve as a source domain for, for example, a concept such as knowledge, the two domains must share the same structure, at least in part. (Lakoff and Johnson, 1980) In other words, 
readers must be able to think and reason about knowledge in the way that they think and reason about columns of water; that is, they must see individuals as containers that are empty until they are filled with liquid-like knowledge; observe that with each layer added, the level of knowledge will go up (i.e., increasing level of knowledge or accumulating knowledge); accept that one can pour knowledge (e.g., into one's mind) but see the layers transferred between persons as discrete volumes in discrete containers (e.g., books, videos, words, and numbers), which are always distinct, irrespective of how tiny they are (e.g., little or no knowledge); view knowledge as a liquid that is possible to pass (on to others), drain (knowledge/brain drain), lose (with death or extinction or just forgotten), and distribute (among others) in the sense that it requires a container in which it can be transferred; observe that people can have different levels of knowledge; and accept that knowledge can have depth (deep knowledge) and extent (extensive knowledge) and that it can overflow and spread. Note, however, that not all aspects of the source domain can be mapped onto the notion of knowledge. For example, the level of knowledge that any teacher might possess does not decrease when some of that knowledge is transferred from one person to another because teachers pass their knowledge on by creating physical expressions in public spaces rather than by transferring it directly from their own minds to those of their students.

The use of the water-handling experience in reasoning about knowledge and many other abstract concepts can be attributed to the conflation of two distinct modes of thinking — one involving nested levels $(((())))$ of the SCALE image schema and the other involving concatenated concrete layers ()()()$($ ) of the ITERATION schema - into a single concept of a water column. Conflation allows the expression of intangible notions (e.g., levels of knowledge) using the more tangible experience of a water column. Diverse ideas, such as comfort, tension, intensity, concentration, significance, eminence, lightness, beauty, pain, strain, effort, change, patience, and magnitude, can all be combined with the term level, which simultaneously imposes a nested and concatenated understanding of those notions. The conflation of levels with layers constitutes a unit that if repeated, gives rise to cognitive products. Such products can then be used to create even larger products, compare experiences, improve the precision of expression, and express the dynamic variation of levels with equivalent steady-state processes, to mention only a few linguistic functions.

A water column is not the only source domain of a relevant embodied experience, and other concepts also share the structure of water columns. The grading systems used in education and elsewhere are one such case, and the 
social concepts of rank, class and hierarchy, currency, numerical systems in mathematics, the act of walking and various measurement scales are others. If a thermometer is used as an example, it is easy to recognize that its scale combines the notion of degree understood as an interval with degrees understood as a nested arrangement, in exactly the same way as water columns combine layers with levels. Understanding this common relationship could facilitate appreciating the difference between the abstract concepts of magnitude, intensity, value, degree, pitch, altitude and quality and the concrete notions of multitude, extent, range, scope, duration, longitude, and quantity. Metaphors draw attention to the close relationship between these abstract notions and their concrete expression. Although they are complex, such relations can be easily experienced and understood by handling containers with water or by recalling this activity from memory. This experience is available to everybody, including young children, and thus, even preschoolers can be introduced to this embodied form of logic on which more complex ideas can be built. Handling water in containers provides a convenient mode of reasoning not only about the structure of linguistic expressions but also about concepts in physics, mathematics and science in general; these subjects will be discussed in separate papers.

\section{References}

APS. 2015. Stress \& Wellbeing: How Australians are Coping with Life. The findings of the Australian Psychological Society Stress and wellbeing in Australia: survey 2015. In Psychology week 8-14 November 2015. https://www.psychology.org.au/Assets/Files/ PW15-SR.pdf accessed on September 9, 2017.

Devlin, Keith. 2008. It Ain't No Repeated Addition. In Devlin's Angle. https://www.maa. org/external_archive/devlin/devlin_06_08.html accessed September 9, 2017.

Estrada-Reynolds, Victoria, Kimberly A. Schweitzer, \& Narina Nuñez. 2016. Emotions in the Courtroom: How Sadness, Fear, Anger, and Disgust Affect Jurors' Decisions, in Wyoming Law Review 343 Volume 16 (2016). Available from http://repository.uwyo .edu/wlr/voli6/iss2/5 accessed on September 9, 2017.

Evans, Vyvyan and Melanie Green. 2006. Cognitive Linguistics: An Introduction. Lawrence Erlbaum Associates, Publishers. London.

Hobson, Ernest William. 1913. Squaring the Circle 'A History of the Problem'. Cambridge: University Press. Accessed on August 19, 2016 from Internet Archive: https://archive.org/stream/squaringthecirclooog63mbp\#page/ng/mode/2up accessed on September 9, 2017 . 
Johnson, Mark. 1987. The Body in the Mind: The Bodily Basis of Meaning, Imagination, and Reason. Chicago: University of Chicago Press.

Kövecses, Zoltán. 2010. Metaphor: A Practical Introduction (second edition). New York: Oxford Press.

Lakoff, George and Mark Johnson. 1980. Metaphors We Live By. London: The University of Chicago Press.

Maranell, Gary M. 2009. Scaling: A Sourcebook for Behavioral Scientists. Transactions Publishing, New Brunswick, New Jersey.

Raykowski, Wes. 2014, Conceptual Understructure of Human Experience: Volume 1 (Thesis). CreateSpace, North Charleston, South Carolina.

Rossi Cesare, Flavio Russo, and Ferruccio Russo. 2009. Ancient engineers' inventions: Precursors of the present. In History of mechanism and machine science Springer, 2009, vol. 8.

Swanson, David. 2009. Help-My Kid is Driving Me Crazy: The 17 Ways Kids Manipulate Their Parents, Victoria, Australia: Penguin Books.

Wiking, Meik (editor). 2014. The Happy Danes: Exploring the reasons behind the high levels of happiness in Denmark. http://jss.org.au/wp-content/uploads/2015/10/ lifesatisfactionreportfinalprintweb.pdf accessed on September 9, 2017.

Williamson, Amelia and Barbara Hoggart. 2005. Pain: a review of three commonly used pain rating scales. In Journal of Clinical Nursing 14, 798-804. 